\title{
Extracción, purificación y cuantificación de mangiferina en la corteza de algunos cultivares de mango (Mangifera indica L.)
}

\section{Extraction, purification and quantification of mangiferin in the bark of some mango (Mangifera indica L.) cultivars}

FREDDY FORERO L. ${ }^{1,3}$

ADRIANA PATRICIA PULIDO D. ${ }^{2}$

Corteza seca del árbol de mango.

Foto: F. Forero L.

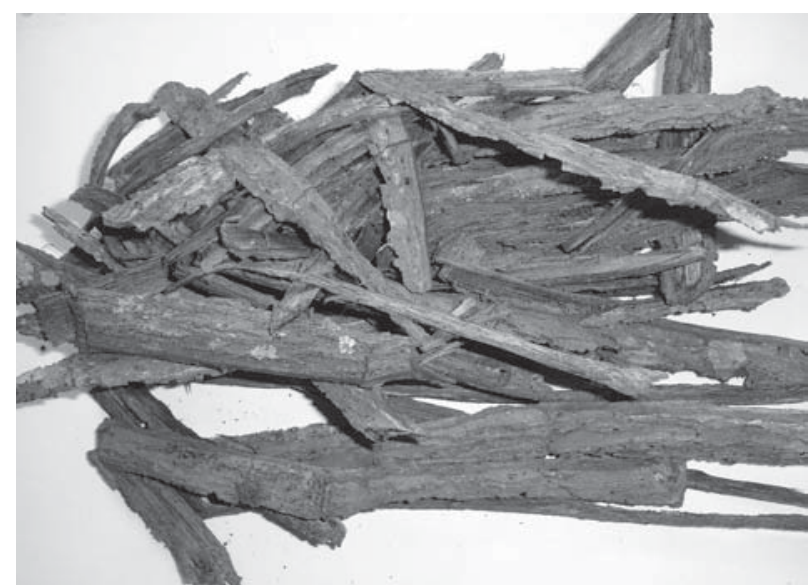

\section{RESUMEN}

La mangiferina $\mathrm{C}_{19} \mathrm{H}_{18} \mathrm{O}_{11}$ (1,3,6,7-Tetrahydroxyxantona-C2- $\beta$-D-glucósido), se aisló a partir de la corteza seca de 40 cultivares del árbol de Mangifera indica L., por extracción líquido/sólido y análisis cuantitativo por espectrofotometría UV-Vis. El producto se caracterizó como un polvo amorfo, amarillo verdoso, soluble en etanol y metanol, punto de fusión $270 \pm 2^{\circ} \mathrm{C}, \lambda_{\max } 370 \mathrm{~nm}$. Los cultivares Pig 693 y Rosa presentan los mayores contenidos del compuesto activo con 5.257,9 \pm 98 y 4.906,2 $\pm 95 \mathrm{mg} / 100 \mathrm{~g}$ bs), los de menor concentración son Bocao e Itamaraca con 698,9 \pm 13 y 893,8 17 (mg/100 g bs), respectivamente. El análisis de conglomerados generó cuatro grupos, donde los mangos asiáticos tuvieron un contenido superior promedio de mangiferina, seguidos por los estadounidenses y los suramericanos. Este trabajo es la primera investigación exploratoria realizada sobre esta xantona en cultivares de mango ubicados en Colombia, sustancia de interés en las áreas de fitomedicina, cosmetología y nutrición.

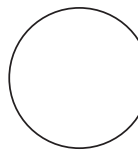

Palabras clave adicionales: Colombia, fitoquímica, metabolitos, xantonas.

\section{ABSTRACT}

The mangiferin $\mathrm{C}_{19} \mathrm{H}_{18} \mathrm{O}_{11}$ (1,3,6,7-Tetrahydroxyxantone-C2- $\beta$-D-glucoside) was isolated from dry bark of 40 cultivars of Mangifera indica L, using liquid/solid extraction and quantitative analysis by UV-Vis spectrophotometry. The product was characterized as an amorphous and greenish yellow powder, soluble in ethanol and

\footnotetext{
Departamento de Alimentos, Universidad de Antioquia, Medellín (Colombia).

2 Escuela Ingeniería de Alimentos, Universidad del Valle, Cali (Colombia).

Autor para correspondencia: freddy.forero@udea.edu.co
} 
methanol, with melting point $270 \pm 2^{\circ} \mathrm{C}, \lambda_{\max } 370 \mathrm{~nm}$. The cultivars Pig 693 and Rosa have the highest content of active compound with $5257,9 \pm 98$ and $4906,2 \pm 95(\mathrm{mg} / 100 \mathrm{~g} \mathrm{db})$, the lower concentrations are in Bocao and Itamaraca with $698,9 \pm 13$ and $893,8 \pm 17 \mathrm{mg} / 100 \mathrm{~g} \mathrm{db}$, respectively. Cluster analysis originated four groups, where Asian mangoes had a higher average content of mangiferin, followed by the North Americans and South Americans. This work is the first exploratory research conducted about this xanthone on mango cultivar located in Colombia, this substance has multiple and effective applications in areas like phytomedicine, cosmetics and nutrition.

Additional key words: Colombia, metabolites, phytochemistry, xanthones.

Fecha de recepción: 06-04-2016 Aprobado para publicación: 24-11-2016

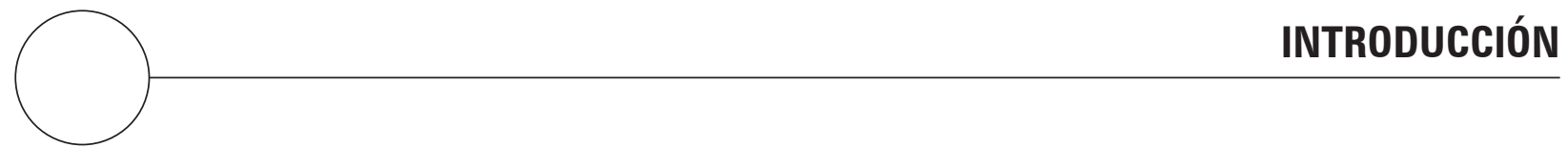

Los estudios relacionados con la extracción y caracterización de mangiferina se remontan a trabajos en la década del sesenta (Bhatia y Seshadri, 1968), quienes aislaron y establecieron la estructura del compuesto, haciendo uso de pruebas selectivas. El producto se obtuvo inicialmente de la madera y corteza del mango (Mangifera indica L.) y fue identificado como un C-glicósido estable del grupo de las xantonas (1,3,6,7-Tetrahydroxyxantona-C2- $\beta$-D-glucósido). La mangiferina es un polifenol que se produce por una ruta biosintética como muestra la de la figura 1 (Joubert et al., 2012; Chavan et al., 2015), está ampliamente distribuida en plantas superiores de las familias Anacardiacae, Gentianaceae y Guttiferae, llamada también una C-glucosilxantona, que se caracteriza por ser resistente a la hidrólisis química y enzimática, a diferencia de las xantonas O-glicósido, en los que el azúcar está unida por un enlace carbono-oxígeno al núcleo y es sensible a la hidrólisis (Scartezzini y Speroni, 2000; Kawakami y Gaspar, 2015). Pocos compuestos pertenecen a este grupo y la mangiferina es la más frecuente, está presente en 60 especies, 18 géneros y 13 familias de dicotiledóneas y se encuentra asociada con C-glucoflavonas, pero no de otras xantonas, por lo que se ha usado como marcador en estudios quimiotaxonómicos (Ferreira et al., 2013; Kulkarni y Rathod, 2015).

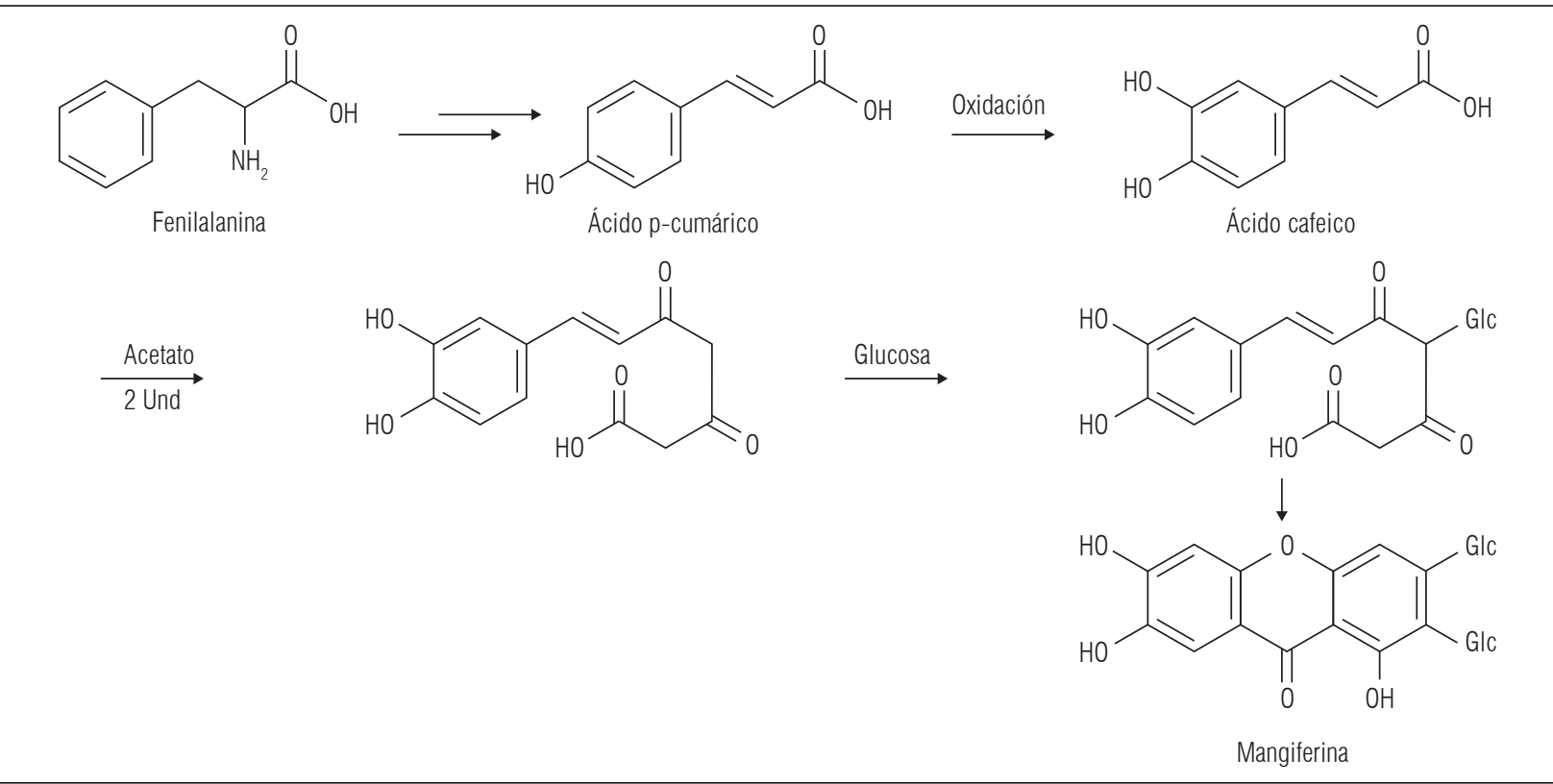

Figura 1. Ruta biosintética para mangiferina en plantas superiores. 
Desde el punto de vista de la extracción se ha observado que las muestras de corteza de mango presentan mayores rendimientos, respecto de la concentración encontrada en las hojas, los rendimientos y la demanda para la industria farmacéutica, ha motivado la búsqueda de nuevas fuentes y se han establecido condiciones para su síntesis, comparando espectroscópicamente la molécula obtenida con el compuesto de origen natural, observando similitud, dejando así las condiciones para la producción a escala de laboratorio (Ruiz-Montañez et al., 2014; Montes et al., 2016). Los C-glucósidos y con ello la mangiferina son fácilmente detectados mediante análisis cromatográfico de extractos vegetales hidrolizados; con el desarrollo de los métodos modernos de análisis se han podido identificar nuevas fuentes y niveles de concentración (Han et al., 2010) (Qiu et al., 2016).

La importancia comercial de mangiferina obedece a su amplio espectro de uso como suplemento nutricional, cosmético y fitomedicinal; en Cuba se desarrolló una decocción acuosa patentada como el producto comercial Vimang ${ }^{\circledR}$ (Beltrán et al., 2004), similar al Salaretin ${ }^{\circledR}$, de Sri Lanka que se usa en los tratamientos de obesidad y diabetes tipo II; se han reportado aplicaciones como agente antiviral, modulador inmunológico y antioxidante (Martínez et al., 2000; Leiro et al., 2003; Mishra et al., 2006; Sethiya et al., 2015). El potencial del banco de germoplasma (colección nacional) de mango localizado en Corpoica (C.I. Nataima) facilita un estudio piloto tendiente a la extracción, purificación y cuantificación de mangiferina presente en la corteza de diversos cultivares de mango, lo cual ayuda a comprender la fisiología y procesos bioquímicos de esta especie a fin de lograr su aprovechamiento con fines científicos y económicos, en el área de los productos naturales y de especial interés para Colombia.

\section{MATERIALES Y MÉTODOS}

\section{Material vegetal}

Las cortezas de Mangifera indica L. (Anacardiaceae) fueron colectadas en la colección nacional de Corpoica, Centro de Investigación Nataima (altitud: 420 msnm, temperatura promedio anual: $28^{\circ} \mathrm{C}$, precipitación promedio anual: $1.400 \mathrm{~mm}$, suelo franco arenoso, en El Espinal, Tolima, Colombia) donde se ha establecido una colección nacional con 86 variedades de mango, de las cuales se escogieron 40 , teniendo en cuenta su importancia comercial, origen, edad y estado fisiológico (Tab. 1).

Las muestras se tomaron de ramas maduras, libres de hongos o enfermedades, retirándolas con un cuchillo de acero inoxidable, evitando cortes de madera; rotulando con fecha, número accesión, variedad, tipo embrionario y origen biogeográfico. Una vez clasificadas, se transportaron al laboratorio donde se pesaron (PE $3600 \pm 0,1 \mathrm{~g}$, Mettler-Toledo International, Greifensee, Suiza) y secaron durante $8 \mathrm{~h}$ a $40 \pm 2^{\circ} \mathrm{C}$ (Hotbox Oven

Tabla 1. Variedades de la colección nacional de mango consideradas en el estudio.

\begin{tabular}{|c|c|c|c|c|c|c|c|c|c|c|c|}
\hline Variedad & $\begin{array}{c}\text { Edad } \\
\text { (años) }\end{array}$ & $\begin{array}{l}\text { Tipo de } \\
\text { embrión }\end{array}$ & Origen & Variedad & $\begin{array}{c}\text { Edad } \\
\text { (años) }\end{array}$ & $\begin{array}{l}\text { Tipo de } \\
\text { embrión }\end{array}$ & Origen & Variedad & $\begin{array}{c}\text { Edad } \\
\text { (años) }\end{array}$ & $\begin{array}{l}\text { Tipo de } \\
\text { embrión }\end{array}$ & Origen \\
\hline Sufaida & 19 & $\mathrm{Me}$ & Asia & Paloma & 19 & $\mathrm{Pe}$ & CYSA & Yulima & 19 & $\mathrm{Me}$ & CYSA \\
\hline Cambodiana & 19 & $\mathrm{Pe}$ & Asia & Albania & 19 & $\mathrm{Pe}$ & CYSA & Bocao R & 19 & $\mathrm{Pe}$ & CYSA \\
\hline Ameery & 19 & Me & Asia & Vallenato & 19 & $\mathrm{Pe}$ & CYSA & Lorito & 19 & $\mathrm{Pe}$ & CYSA \\
\hline Mulgoba & 16 & Me & Asia & Azúcar & 18 & $\mathrm{Pe}$ & CYSA & ICA 1831 & 19 & $\mathrm{Pe}$ & CYSA \\
\hline Pig 693 & 19 & $\mathrm{Me}$ & Asia & Bocao & 18 & $\mathrm{Pe}$ & CYSA & Durazno & 19 & $\mathrm{Pe}$ & CYSA \\
\hline Bombay Yelow & 19 & Me & Asia & Maza & 18 & $\mathrm{Pe}$ & CYSA & Itamaraca & 19 & $\mathrm{Pe}$ & CYSA \\
\hline Irwin & 19 & Me & EU & Corazón & 18 & $\mathrm{Pe}$ & CYSA & Manila & 19 & $\mathrm{Pe}$ & Asia \\
\hline Vandyke & 19 & Me & EU & Piedra & 18 & $\mathrm{Pe}$ & CYSA & Uba & 19 & $\mathrm{Pe}$ & Asia \\
\hline Tommy Atkins & 19 & $\mathrm{Me}$ & EU & Chucunda & 17 & $\mathrm{Me}$ & CYSA & Filipino & 19 & $\mathrm{Pe}$ & Asia \\
\hline Kent & 17 & Me & EU & Manzano V & 17 & $\mathrm{Pe}$ & CYSA & Early Gold & 19 & $\mathrm{Pe}$ & EU \\
\hline Zill & 17 & $\mathrm{Me}$ & EU & Chancleto & 17 & $\mathrm{Pe}$ & CYSA & Keitt & 19 & $\mathrm{Me}$ & EU \\
\hline Carrie & 17 & Me & EU & Mariquita & 18 & $\mathrm{Pe}$ & CYSA & Chino & 16 & $\mathrm{Pe}$ & CYSA \\
\hline Julie & 19 & $\mathrm{Me}$ & EU & Rosa & 18 & $\mathrm{Pe}$ & CYSA & & & & \\
\hline Edward & 19 & $\mathrm{Me}$ & EU & ICA 1839 & 17 & $\mathrm{Pe}$ & CYSA & & & & \\
\hline
\end{tabular}

Me: monoembrionico; Pe: poliembrionico; EU: Estados Unidos; CYSA: Centro y Suramérica. 
size 1, Gallenkamp) (Lab Merchant, London). La corteza seca (CS) fue molida a un tamaño de partícula de $2 \mathrm{~mm}$ (Lab. Mill model 4, Thomas-Wiley, Swedesboro, $\mathrm{NJ}$ ) para finalmente ser empacadas en bolsas al vacíoy almacenadas bajo congelación $\left(-15 \pm 1^{\circ} \mathrm{C}\right)$.

\section{Extracción}

Se aplicó un protocolo base (Núñez et al., 2002), con ligeras modificaciones; corteza seca y molida (50 g) por triplicado, se sometió a extracción líquido/sólido en unidad Soxhlet $(250 \mathrm{~mL})$, para remoción de la fracción grasa con éter de petróleo 40:60 $(250 \mathrm{~mL} \times 8$ h), posteriormente con $\mathrm{Me}_{2} \mathrm{CO}(250 \mathrm{~mL} \times 8 \mathrm{~h})$, para eliminación de taninos y finalmente con $\mathrm{EtOH} / \mathrm{H}_{2} \mathrm{O}$ $(70: 30 \mathrm{v} / \mathrm{v})(250 \mathrm{~mL} \times 8 \mathrm{~h})$ para la obtención del extracto total (ET), que fue concentrado a $150 \mathrm{~mL}$ en un rotovapor (Laborota 4011, Heidolph Instruments, Schwabach, Alemania) con posterior almacenamiento a $-15 \pm 1^{\circ} \mathrm{C}$ y protegidos de la luz.

\section{Purificación y caracterización}

La mangiferina patrón se obtuvo usando una muestra de ET, filtrando al vacío (Vacuum Station, Barnant, Barrington, IL), empleando una membrana de $7 \mu \mathrm{m}$ (Whatman GF/C), el ET filtrado fue concentrado y la mangiferina fue recristalizada en $\mathrm{EtOH} 96 \%$ bajo presión reducida y recuperada como un sólido cristalino que fue almacenado en frascos ámbar bajo temperatura de congelación $\left(-15 \pm 1^{\circ} \mathrm{C}\right)$. Al producto patrón se le determinó punto de fusión (M5000, Krüss), rotación óptica (P1000-LED, Krüss) y análisis por espectrofotometría UV-Vis (EtOH) (Helios Zeta, Thermo Scientific: Thomas Scientific, Swedesboro, NJ) con un barrido en el rango 200 a $650 \mathrm{~nm}$, evaluando el comportamiento ácido-base, para establecer los desplazamientos a máxima absorbancia, la información se verificó y complementó con datos reportados en la literatura científica.

\section{Cuantificación y análisis estadístico}

La cuantificación de la mangiferina en los ET, se realizó mediante espectrofotometría UV-VIS, a una longitud de onda de $370 \mathrm{~nm}$, para lo cual se preparó una curva patrón de mangiferina en EtOH/ $\mathrm{H}_{2} \mathrm{O}$ (70:30 $\mathrm{v} / \mathrm{v}$ ), usando la muestra previamente recristalizada y caracterizada, las concentraciones utilizadas fueron 1, 5, 10, 20, 30, 50, 70 ppm de mangiferina, cada concentración fue preparada por triplicado. Una vez construida la curva de calibración, la determinación del contenido de mangiferina se realizó sobre los ET homogenizados y atemperados, tomando un volumen de $3 \mu \mathrm{L}$ (Micropipette 841, Socorex, Suiza), completando a $1 \mathrm{~mL}$ con $\mathrm{EtOH} / \mathrm{H}_{2} \mathrm{O}(70: 30 \mathrm{v} / \mathrm{v})$ en una celda de cuarzo y leyendo los valores de absorbancia a $370 \mathrm{~nm}$, previa calibración del equipo con un blanco $\mathrm{EtOH} 96 \%$, los resultados finales se expresaron en $\mathrm{mg}$ de mangiferina por $100 \mathrm{~g}$ CS.

Todos los datos generados se analizaron con el paquete estadístico Statgraphics 5 Plus ${ }^{\circledR}$ (StatPoint Inc.), efectuando pruebas intra e intervariedades para verificar si existían diferencias significativas (95\% confianza) para el contenido de mangiferina, con el fin de identificar posibles relaciones multivariantes de agrupación se utilizó el análisis de conglomerados para clasificar a un conjunto de individuos (variedades) en grupos homogéneos, el método usado fue el de Ward, aplicando la distancia métrica euclidiana.

\section{RESULTADOS Y DISCUSIÓN}

\section{Material vegetal y extracción}

Se evidenció que la etapa de molido tiene especial importancia, ya que el tamaño de partícula elegido fue limitante, con tamaño menor a $2 \mathrm{~mm}$ se formó una masa semisólida, que dificultó la recirculación y penetración del solvente. La eliminación de lípidos y taninos facilita en gran medida el proceso de cuantificación de la mangiferina, de no ser así, estos compuestos serían arrastrados por el EtOH, debido a un efecto de cosolvencia, lo cual provoca un error significativo al interferir en la banda de absorbancia máxima.

El uso de EtOH en la etapa final está justificado por ser uno de los solventes menos costosos, tener una toxicidad muy baja y permitir la extracción a temperatura menor de $80^{\circ} \mathrm{C}$, limitando la formación de compuestos no deseados, la principal ventaja del uso de mezclas de solventes en procesos de extracción reside en la capacidad de incremento de la relación de polaridades que contribuyó de manera selectiva a la eficiencia de la extracción, la polaridad estimada para la mezcla $\mathrm{EtOH} / \mathrm{H}_{2} \mathrm{O}(70: 30 \mathrm{v} / \mathrm{v})$ fue de 63,4 más baja que el agua (80) pero mayor el etanol $(24,6)$ (Faizi et al., 2006). 


\section{Caracterización}

El compuesto patrón de mangiferina obtenido se caracterizó como un polvo amorfo de color amarillo verdoso, soluble en $\mathrm{EtOH}$ y $\mathrm{MeOH}$; presentó punto de fusión de $270 \pm 0,5^{\circ} \mathrm{C}$, y rotación específica $[\alpha]^{25}$ $\geq 34^{\circ}$, el espectro UV-VIS (Fig. 2) presentó señales de máxima absorbancia a 259, 316 y $370 \mathrm{~nm}$ que son coincidentes con moléculas tipo xantona y en particular con la mangiferina.

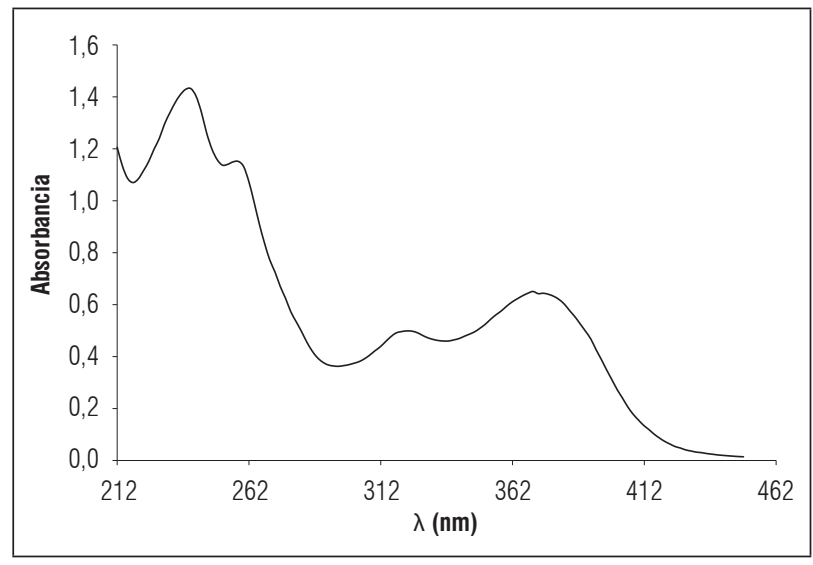

Figura 2. Espectro UV-Vis (EtOH) del patrón de mangiferina obtenido de la corteza del árbol de mango.

Se observaron cuatro bandas de absorción principales a $\lambda_{\max } 240 \mathrm{~nm}$ de transiciones electrónicas $\pi \rightarrow^{*} \pi$ del núcleo aromático, la segunda entre 230 y $260 \mathrm{~nm}$ debida a la transición $\pi \rightarrow^{*} \pi$ de uno de los anillos continuos al núcleo central que contiene el enlace carbonilo, la tercera banda de menor intensidad con un máximo a $317 \mathrm{~nm}$ relacionada con la transición $\mathrm{n} \rightarrow \pi^{*}$ del anillo aromático, la última banda con $\lambda_{\max }$
$370 \mathrm{~nm}$ cercana al rango visible, podría ser asignada a la transferencia de carga intermolecular (Gómez-Zaleta et al., 2006) .

La molécula de mangiferina extraída, presentó un marcado comportamiento ácido-base, los cambios en el $\mathrm{pH}$ del solvente indujeron desplazamientos a longitudes de onda mayor (efecto batocrómico). A pH 4,0, la primera banda de absorción presentó un efecto hipercrítico, seguido por un efecto hipocrómico, después de alcanzar un $\mathrm{pH}$ cercano a 7,6 que se acompaña de un marcado efecto batocrómico de 257 a 271 nm que puede estar relacionado a la formación de un ion enolato y extinción de la tercera banda; la última banda alcanza su máximo de absorción a pH 9,6 con $(390 \mathrm{~nm})$, con un pronunciado efecto hipocrómico (Fig. 3), este comportamiento es similar al reportado por otros autores (Mishra et al., 2006).

La fracción mayoritaria de los extractos blandos obtenidos de las cortezas, está conformada por polifenoles, donde se destaca la presencia de mangiferina como el componente mayoritario. De su estructura química, se concluye que la mangiferina cumple con los cuatro requisitos descritos en la literatura científica para lograr la mejor biodisponibilidad por vía oral: (1) peso molecular menor de 500, (2) menos de cinco funciones donantes para enlaces de hidrógeno, (3) menos de 10 funciones aceptoras de enlaces de hidrógeno, (4) $\log \mathrm{P}$ (potencial) calculado menor de +5 $\left(\log _{\mathrm{P}}\right.$ mangiferina $\left.=+2,73\right)$ (Beltrán et al., 2004).

\section{Cuantificación}

La determinación cuantitativa procedió luego de establecer la linealidad de la curva de calibración,

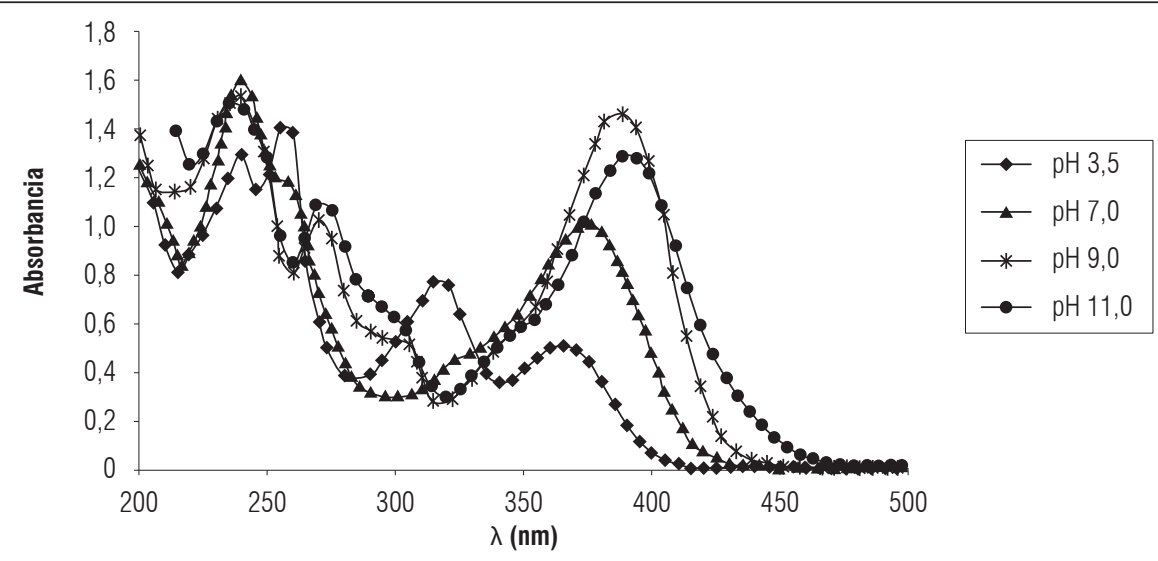

Figura 3. Comportamiento ácido base de la mangiferina entre pH 3,5 y 11,0. 
el coeficiente de correlación fue de $99,92 \%\left(r^{2}=\right.$ 0,9992), el rango dinámico de respuesta varió entre 0,232 a 0,975 unidades de absorbancia, equivalente a 0,005-0,020 mg mag/mL, para los límites de detección mínimo y máximo, respectivamente, los cuales estuvieron dentro de los parámetros analíticos reportados en otras investigaciones (Ruiz-Montañez et al., 2014).

Los datos para los contenidos totales de mangiferina presentaron una distribución normal, la cantidad de mangiferina extraída se ve afectada por la variedad $(P \leq 0,05)$, por lo que se procedió a efectuar la prueba de rangos múltiples (Tukey HSD) para encontrar qué variedades se agrupaban en grupos homogéneos (Tab. 2). Los cultivares Pig 693, Rosa y Julie, fueron los dos grupos con mayor contenido de mangiferina con $5.257,4.906,2$ y $4.879,1 \mathrm{mg} \mathrm{mag} / 100 \mathrm{~g}$ CS, respectivamente, caso contrario se observó en los materiales Bocao $(698,9)$ e Itamaraca $(893,8)$ que tienen los contenidos de mangiferina más bajos. Esta marcada diferencia puede ser determinada por factores genéticos, ontogenéticos y ambientales, pues la producción de compuestos bioactivos se da a nivel secundario del metabolismo y este a su vez es una función de la expresión génica (Ribeiro et al., 2008).

Al efectuar el análisis de conglomerados (Fig. 4), teniendo como variables de agrupación la variedad, origen, tipo de embrión y contenido de mangiferina, se identificaron cuatro grupos claramente diferenciados, donde se aprecia que los factores origen y contenido de mangiferina fueron los de mayor peso en el proceso de agrupación.

En el conglomerado 1 se encuentran ubicados las variedades de Centro y Suramérica (CYSA) caracterizados por ser en su mayoría (90\%) del tipo poliembriónico (Pe) y presentar el nivel promedio más bajo en el contenido de mangiferina; los mangos de Asia se ubicaron en el conglomerado 2 con una distribución

Tabla 2. Rendimiento de extracto blando (EB) y contenido de mangiferina en la corteza seca (CS) de cultivares de mango ( $n=3$, \pm desviación estándar).

\begin{tabular}{|c|c|c|c|c|c|}
\hline \multirow{2}{*}{ Variedad } & Extracto blando & Contenido mangiferina & \multirow{2}{*}{ Variedad } & Extracto blando & Contenido mangiferina \\
\hline & g EB/100 g CS & $\mathrm{mg}$ mag $/ 100 \mathrm{~g} \mathrm{CS}$ & & $\mathrm{g} \mathrm{EB} / 100 \mathrm{~g} \mathrm{CS}$ & $\mathrm{mg}$ mag $/ 100 \mathrm{~g} \mathrm{CS}$ \\
\hline Itamaraca & $18,4 \pm 0,7$ & $893,8 \pm 17 b$ & Chucunda & $19,2 \pm 0,7$ & $1.246,1 \pm 24 \mathrm{c}, \mathrm{d}$ \\
\hline Bocao & $9,2 \pm 0,3$ & $698,9 \pm 13 a$ & Kent & $11,6 \pm 0,4$ & $1581,7 \pm 31 \mathrm{~g}$ \\
\hline Lorito & $17,2 \pm 0,6$ & $939,2 \pm 18 b$ & Zill & $17,6 \pm 0,7$ & $2.009,3 \pm 40 \mathrm{k}, \mathrm{l}, \mathrm{m}, \mathrm{n}$ \\
\hline Sufaida & $58,4 \pm 2,3$ & $2.656,0 \pm 53 q$ & Vallenato & $14 \pm 0,5$ & $1.814,4 \pm 36 h, \mathrm{i}$ \\
\hline Yulima & $17,6 \pm 0,7$ & $1.147,1 \pm 22 c$ & Chancleto & $12,8 \pm 0,5$ & $1.132,5 \pm 22 c$ \\
\hline ICA 1831 & $12 \pm 0,4$ & $1.333,8 \pm 26 \mathrm{~d}, \mathrm{e}$ & Carrie & $33,2 \pm 1,3$ & $1.457,2 \pm 29 \mathrm{e}, \mathrm{f}, \mathrm{g}$ \\
\hline Durazno & $22,8 \pm 0,9$ & $2.095,8 \pm 41$ I,m,n,0 & Early Gold & $9,2 \pm 0,3$ & $1.489,7 \pm 29 \mathrm{f}, \mathrm{g}$ \\
\hline Paloma & $6,8 \pm 0,2$ & $1.341,9 \pm 26 \mathrm{~d}, \mathrm{e}, \mathrm{f}$ & Pig 693 & $26,4 \pm 1,0$ & $5.257,9 \pm 98 v$ \\
\hline Manila & $13,6 \pm 0,5$ & $1.544,9 \pm 30 \mathrm{~g}$ & Julie & $24,8 \pm 0,9$ & $4.879,1 \pm 97 u$ \\
\hline Irwin & $16,4 \pm 0,6$ & $1.906,4 \pm 38$ h,i,j,k & Edward & $11,2 \pm 0,4$ & $1.538,4 \pm 30 \mathrm{~g}$ \\
\hline Vandyke & $14,8 \pm 0,5$ & $1.971,4 \pm 39 \mathrm{j}, \mathrm{k}, \mathrm{l}, \mathrm{m}$ & Bombay & $12,4 \pm 0,4$ & $2.122,9 \pm 42 m, n, 0$ \\
\hline Albania & $23,2 \pm 0,9$ & $2.561,3 \pm 51 p, q$ & Uba & $18,4 \pm 0,7$ & $2.139,1 \pm 45 n, 0$ \\
\hline Tommy Atkins & $15,6 \pm 0,6$ & $2.574,8 \pm 51 p, q$ & Keitt & $17,2 \pm 0,6$ & $3.531,4 \pm 70 r$ \\
\hline Manzano & $14,4 \pm 0,5$ & $1.976,8 \pm 39 h, i$ & Ameery & $25,2 \pm 1,0$ & $4.716,7 \pm 94 \mathrm{t}$ \\
\hline Cambodiana & $24,4 \pm 0,9$ & $1.511,3 \pm 30 \mathrm{f}, \mathrm{g}$ & Mariquita & $12 \pm 0,4$ & $1.955,1 \pm 39 \mathrm{i}, \mathrm{j}, \mathrm{k}, \mathrm{l}$ \\
\hline Azúcar & $11,2 \pm 0,4$ & $2.187,9 \pm 430$ & Rosa & $13,2 \pm 0,5$ & $4.906,2 \pm 95 u$ \\
\hline Bocao reina & $22 \pm 0,8$ & $2.439,5 \pm 48 p$ & ICA 1839 & $29,6 \pm 1,1$ & $2.439,5 \pm 48 p$ \\
\hline Maza & $14,8 \pm 0,5$ & $1.516,7 \pm 30 \mathrm{~g}$ & Chino & $22,4 \pm 0,8$ & $1.560,0 \pm 31 \mathrm{~g}$ \\
\hline Corazón & $19,2 \pm 0,7$ & $2.068,8 \pm 411, m, n, 0$ & Mungoba & $17,2 \pm 0,6$ & $1.841,5 \pm 36$ h,i,j \\
\hline Piedra & $8,8 \pm 0,3$ & $1.798,2 \pm 35 \mathrm{~h}$ & Filipino & $16,8 \pm 0,6$ & $4.191,7 \pm 83 \mathrm{~s}$ \\
\hline
\end{tabular}

Promedios con letras distintas indican diferencia significativa según la prueba de Tukey $(P \leq 0,05)$. 


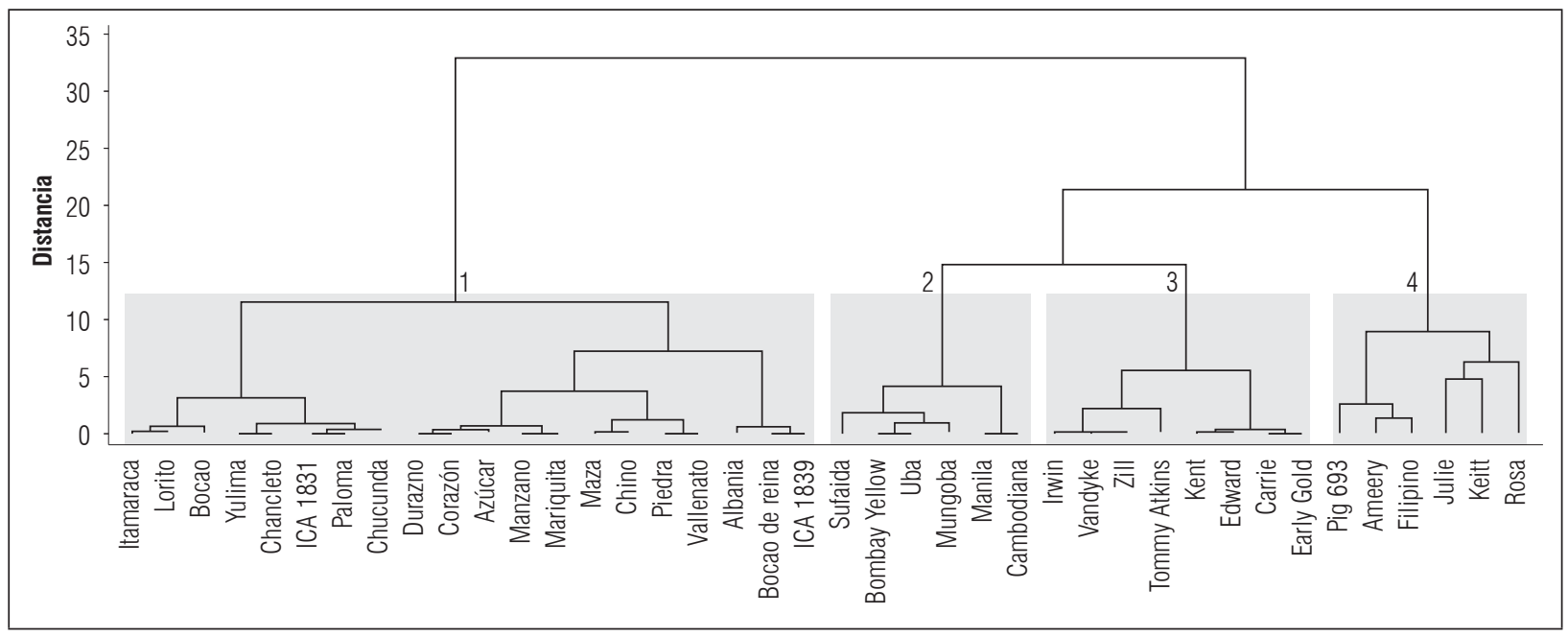

Figura 4. Dendrograma para el análisis de conglomerados entre las variedades de mango, origen, tipo de embrión y contenido de mangiferina.

homogénea (50:50) para el tipo de embrión y un contenido de mangiferina alto; las variedades de Estados Unidos fueron $88 \%$ monoembriónicas y con un contenido de mangiferina medio, siendo todas ellas pertenecientes al grupo 3, finalmente el conglomerado 4 lo componen 6 variedades de orígenes mixtos y con muy altos contenidos del compuesto activo mangiferina.

Es necesario tener en cuenta el factor ontogenético, porque la concentración de compuestos bioactivos como la mangiferina varió de acuerdo al origen y desarrollo de la planta, en ese sentido, las variedades autóctonas (CYSA), con escasa importancia económica y no sometidas a procesos de mejoramiento, podrían ser una explicación para presentar un menor contenido de compuestos bioactivos (Kulkarni y Rathod,
2014). Por otro lado, los factores ambientales pueden modificar la producción de metabolitos secundarios, influenciando directamente la expresión de los genes, en ese sentido, los genes responsables para la producción de metabolitos se pueden activar o desactivar de acuerdo con las condiciones climáticas o nutricionales, ataque de plagas (Ribeiro et al., 2008).

La identificación de las variedades con altos contenidos de mangiferina es un resultado importante desde el punto de vista tecnológico, teniendo en cuenta que los subproductos, como la corteza, son generalmente desechados durante las labores de mantenimiento del cultivo de mango, siendo posible utilizarlos como materia prima para obtener compuestos de alto valor agregado y disminuir simultáneamente posibles contaminantes ambientales.

Tabla 3. Resumen paramétrico del análisis de conglomerados entre las variedades de mango, origen, tipo de embrión y contenido de mangiferina.

\begin{tabular}{|c|c|c|c|c|c|c|c|}
\hline \multirow{2}{*}{ Conglomerado } & \multirow{2}{*}{ Origen } & \multirow{2}{*}{ Miembros } & \multicolumn{3}{|c|}{ Tipo de embrión } & \multicolumn{4}{c|}{ Mangiferina } \\
\cline { 5 - 8 } & & & Me & Pe & Máximo & Mínimo & Media \\
\hline 1 & CYSA & 20 & 2 & 18 & $2.561,3$ & 698,9 & $1.657,4$ \\
\hline 2 & Asia & 6 & 3 & 3 & $2.656,0$ & $1.511,3$ & $1.969,3$ \\
\hline \multirow{2}{*}{3} & EU & 8 & 7 & 1 & $2.574,8$ & $1.457,2$ & $1.816,1$ \\
\cline { 2 - 8 } & Asia & 3 & 2 & 1 & $5.257,9$ & $4.191,7$ & $4.722,1$ \\
\hline \multirow{2}{*}{4} & EU & 2 & 2 & 0 & $4.879,1$ & $3.531,4$ & $4.205,3$ \\
\cline { 2 - 8 } & CYSA & 1 & 0 & 1 & $5.004,2$ & $4.808,2$ & $4.906,2$ \\
\hline
\end{tabular}

Me: monoembriónico; Pe: poliembrionico; EU: Estados Unidos; CYSA: Centro y Suramérica. 


\section{CONCLUSIONES}

Los métodos empleados para la extracción, purificación y cuantificación de la mangiferina fueron adecuados, encontrándose que la corteza del árbol de mango es una gran fuente de este metabolito secundario, se identificaron diferencias significativas intra e intergrupos para el contenido de mangiferina, encontrándose a las variedades Pig 693 y Rosa, como las de mayor concentración de mangiferina, la limitación en el uso de estas variedades podría ser la poca área plantada a nivel comercial. Se identificó una gran variabilidad en los contenidos de mangiferina que puede ser explicada por el cruzamiento de manera natural, formando un complejo de híbridos interraciales e intraraciales, los cuales han dado origen a más de un millar de variedades de mango. El procedimiento presentado $y$ variedades promisorias es una iniciativa preliminar que puede ser evaluada a nivel de laboratorio y posteriormente en planta piloto con fines de comercialización, en este sentido habría que considerarse la recuperación de los solventes, lo cual se reducirá el posible impacto ambiental y costos de producción.

\section{AGRADECIMIENTOS}

Los autores expresan su agradecimiento a la Corporación Colombiana de Investigación Agropecuaria (Corpoica, Centro de Investigación Nataima), por su apoyo en la consecución del material vegetal y logística.

\section{REFERENCIAS BIBLIOGRÁFICAS}

Beltrán, A.E., Y. Álvarez, F. E. Xavier, R. Hernanz, J. Rodríguez, A.J. Núñez, M.J. Alonso y M. Salaices. 2004. Vascular effects of the Mangifera indica L. extract (Vimang). Eur. J. Pharmacol. 499(3), 297-305. Doi: 10.1016/j.ejphar.2004.07.073

Bhatia, V.K. y T.R. Seshadri. 1968. Synthesis of mangiferin. Tetrahedron Lett. 9(14), 1741-1742. Doi: 10.1016/ S0040-4039(01)99040-9

Chavan, J.J., D.M. Ghadage, A.S. Bhoite y S.D. Umdale. 2015. Micropropagation, molecular profiling and RPHPLC determination of mangiferin across various regeneration stages of Saptarangi (Salacia chinensis L.). Ind. Crops Prod. 76, 1123-1132. Doi: 10.1016/j. indcrop.2015.08.028

Faizi, S., S. Zikr-ur-Rehman, M. Ali y A. Naz. 2006. Temperature and solvent dependent NMR studies on mangiferin and complete NMR spectral assignments of its acyl and methyl derivatives. Magn. Resonance Chem. 44(9), 838-844. Doi: 10.1002/mrc.1854
Ferreira, F.d.R., I.B. Valentim, E.L.C. Ramones, M.T.S. Trevisan, C. Olea-Azar, F. Perez-Cruz, F.C. de Abreu y M.O.F. Goulart. 2013. Antioxidant activity of the mangiferin inclusion complex with $\beta$-cyclodextrin. LWT-Food Sci. Technol. 51(1), 129-134. Doi: 10.1016/j. lwt.2012.09.032

Gómez-Zaleta, B., M.T. Ramírez-Silva, A. Gutiérrez, E. González-Vergara, M. Güizado-Rodríguez y A. Rojas-Hernández. 2006. UV/vis, 1H, and 13C NMR spectroscopic studies to determine mangiferin $\mathrm{pKa}$ values. Spectrochimica Acta Part A: Mol. Biomol.Spectrosc. 64(4), 1002-1009. Doi: 10.1016/j.saa.2005.09.009

Han, D., C. Chen, C. Zhang, Y. Zhang y X. Tang. 2010. Determination of mangiferin in rat plasma by liquid-liquid extraction with UPLC-MS/MS. J. Pharm. Biomed. Anal. 51(1), 260-263. Doi: 10.1016/j. jpba.2009.07.021

Joubert, E., M. Botha, C. Maicu, D. De Beer y M. Manley. 2012. Rapid screening methods for estimation of mangiferin and xanthone contents of Cyclopia subternata plant material. South Afr. J. Bot. 82, 113-122. Doi: 10.1016/j.sajb.2012.07.019

Kawakami, C.M. y L.R. Gaspar. 2015. Mangiferin and naringenin affect the photostability and phototoxicity of sunscreens containing avobenzone. J. Photochem. Photobiol. B: Biol. 151, 239-247. Doi: 10.1016/j. jphotobiol.2015.08.014

Kulkarni, V.M. y V.K. Rathod. 2014. Extraction of mangiferin from Mangifera indica leaves using three phase partitioning coupled with ultrasound. Ind. Crops Prod. 52, 292-297. Doi: 10.1016/j.indcrop.2013.10.032

Kulkarni, V.M. y V.K. Rathod. 2015. A novel method to augment extraction of mangiferin by application of microwave on three phase partitioning. Biotechnol. Rep. 6, 8-12. Doi: 10.1016/j.btre.2014.12.009

Leiro, J.M., E. Álvarez, J.A. Arranz, I.G. Siso y F. Orallo. 2003. In vitro effects of mangiferin on superoxide concentrations and expression of the inducible nitric oxide synthase, tumour necrosis factor- $\alpha$ and transforming growth factor- $\beta$ genes. Biochem. Pharm. 65(8), 1361-1371. Doi: 10.1016/S0006-2952(03)00041-8

Martínez, G., R. Delgado, G. Pérez, G. Garrido, A.J. Núñez S. y O.S. León. 2000. Evaluation of the in vitro antioxidant activity of Mangifera indica L. extract (Vimang). Phytother. Res. 14(6), 424-427. Doi: 10.1002/10991573(200009)14:6<424::AID-PTR643>3.0.CO;2-8

Mishra, B., K.I. Priyadarsini, M. Sudheerkumar, M.K. Unnikrishhnan y H. Mohan. 2006. Pulse radiolysis studies of mangiferin: A C-glycosyl xanthone isolated from Mangifera indica. Rad. Phys. Chem. 75(1), 70-77. Doi: 10.1016/j.radphyschem.2005.05.015

Montes, A., L. Wehner, C. Pereyra y E.J.M. Ossa. 2016. Mangiferin nanoparticles precipitation by supercritical 
antisolvent process. J. Supercrit. Fluids 112, 44-50. Doi: 10.1016/j.supflu.2016.02.008

Núñez S., A.J., H.T. Vélez C., J. Agüero-Agüero, J. González-González, F. Naddeo, F. De Simone y L. Rastrelli. 2002. Isolation and quantitative analysis of phenolic antioxidants, free sugars, and polyols from mango (Mangifera indica L.) stem bark aqueous decoction used in Cuba as a nutritional supplement. J. Agric. Food Chem. 50(4), 762-766. Doi: 10.1021/jf011064b

Qiu, X., J.-1. Zhao, C. Hao, C. Yuan, N. Tian, Z.-S. Xu y R.M. Zou. 2016. Simultaneous determination of mangiferin and neomangiferin in rat plasma by UPLC-MS/ MS and its application for pharmacokinetic study. J. Pharm. Biomed. Anal. 124, 138-142. Doi: 10.1016/j. jpba.2016.02.034

Ribeiro, S.M.R., L.C.A. Barbosa, J.H. Queiroz, M. Knödler y A. Schieber. 2008. Phenolic compounds and antioxidant capacity of Brazilian mango (Mangifera indica L.) varieties. Food Chem. 110(3), 620-626. Doi: 10.1016/j. foodchem.2008.02.067

Ruiz-Montañez, G., J.A. Ragazzo-Sánchez, M. Calderón-Santoyo, G. Velázquez-de la Cruz, J.A. Ramírez de León y A. Navarro-Ocaña. 2014. Evaluation of extraction methods for preparative scale obtention of mangiferin and lupeol from mango peels (Mangifera indica L.). Food Chem. 159, 267-272. Doi: 10.1016/j. foodchem.2014.03.009

Scartezzini, P. y E. Speroni. 2000. Review on some plants of Indian traditional medicine with antioxidant activity. J. Ethnopharm. 71(1-2), 23-43. Doi: 10.1016/ S0378-8741(00)00213-0

Sethiya, N.K., A. Trivedi y S.H. Mishra. 2015. Rapid validated high performance thin layer chromatography method for simultaneous estimation of mangiferin and scopoletin in Canscora decussata (South Indian Shankhpushpi) extract. Rev. Bras. Farmacognosia 25(3), 193 198. Doi: 10.1016/j.bjp.2015.04.002 